\title{
Topological insulator on the kagome lattice
}

\author{
H.-M. Guo and M. Franz \\ Department of Physics and Astronomy, University of British Columbia, Vancouver, BC, Canada V6T $1 Z 1$
}

(Dated: September 25, 2009)

\begin{abstract}
Itinerant electrons in a two-dimensional kagome lattice form a Dirac semi-metal, similar to graphene. When lattice and spin symmetries are broken by various periodic perturbations this semi-metal is shown to spawn interesting non-magnetic insulating phases. These include a twodimensional topological insulator with a non-trivial $\mathrm{Z}_{2}$ invariant and robust gapless edge states, as well as dimerized and trimerized 'Kekulé' insulators. The latter two are topologically trivial but the Kekulé phase possesses a complex order parameter with fractionally charged vortex excitations. A charge density wave is shown to couple to the Dirac fermions as an effective axial gauge field.
\end{abstract}

Certain physical observables in solids, such as the magnetic flux in a superconductor or the Hall conductance in a quantum Hall liquid, are precisely quantized despite the fact that the host material may contain a significant amount of disorder. In all known cases this quantization phenomenon can be attributed to the notion of topological order. The bulk of such systems are characterized by a topological invariant that is insensitive to microscopic details and robust with respect to weak disorder. Recently, a new class of topological invariants has been established to characterize all time-reversal $(\mathcal{T})$ invariant band insulators in 2 and 3 spatial dimensions [1, 2, 3, 4]. These new invariants are of the $\mathrm{Z}_{2}$ variety and the precisely quantized physical observable is the number of gapless edge (surface) states modulo 2. Topological insulators (TI) exhibit an odd number of edge (surface) states while trivial insulators exhibit an even number, possibly zero. In many ways the edge (surface) states of a TI behave as a perfect metal and are predicted to exhibit various unusual properties [5, 6, 7. They also show promise as possible components of future quantum computers 8 .

Experimentally, $\mathrm{HgTe} /(\mathrm{Hg}, \mathrm{Cd}) \mathrm{Te}$ quantum wells of certain width and composition have been identified as 2D topological 'spin Hall' insulators with robust edge states [9]. In addition, several 3D compounds involving bismuth have been so identified [10, 11] and several more have been predicted 12, 13 as likely candidates. In view of these rapid developments it appears likely that TIs might be a fairly common occurrence in nature. Given their exotic properties and their potential for technological applications it is important to identify and study various model systems that exhibit this behavior. Such theoretical understanding will aid experimental searches for new materials and help understand their unusual properties.

In this Brief Report we advance the above agenda by describing a new class of 2-dimensional topological insulators on the kagome lattice, Fig. 17. Although the properties of spin systems on the kagome lattice have been extensively studied, relatively little attention has been paid to the non-magnetic insulating phases of itinerant electrons. In what follows we demonstrate, both analytically and numerically, that a simple tight-binding model of electrons on the kagome lattice at both $\frac{1}{3}$ and $\frac{2}{3}$ filling becomes a topological insulator upon inclusion
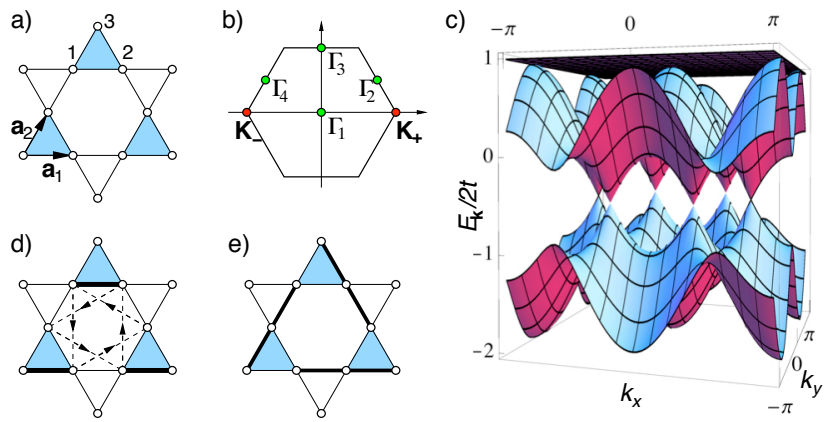

FIG. 1: (Color online) a) kagome lattice is a triangular Bravais lattice with a 3-point basis forming a shaded triangle. b) The first Brillouin zone with the nodal points $\mathbf{K}_{ \pm}$and timereversal invariant momenta $\boldsymbol{\Gamma}_{n}$ marked. c) The tight-binding band structure Eq. (3). d) In the dimerized phase hopping amplitude along the thick (thin) bonds in $\mathbf{a}_{1}$ direction is $t+\eta_{1}$ $\left(t-\eta_{1}\right)$. In the spin-orbit phase spin-up electrons hop between second neighbor sites with amplitude $i \lambda$ when moving along the arrow, $-i \lambda$ against the arrow. For spin-down electrons the arrows are reversed. e) Trimerized 'Kekulé' phase.

of the spin-orbit (SO) coupling. Other $\mathcal{T}$-invariant insulating phases include the dimerized and a trimerized band insulators; both have trivial $\mathrm{Z}_{2}$ invariants but the latter possesses a complex order parameter with vortices that carry fractional charge, in analogy to what happens in the Kekulé phase of graphene [14]. We demonstrate that a charge density wave (CDW) modulation of the on-site energies does not produce a spectral gap but instead generates terms that couple as a U(1) gauge field to the low-energy Dirac fermions at $\frac{1}{3}$ filling. Consistent with $\mathcal{T}$-invariance, the gauge field couples with opposite sign to the two species of Dirac fermions, thus furnishing a concrete realization of an axial gauge field in a solid state system.

We now proceed to substantiate the above claims. Our starting point is the tight-binding model

$$
H_{0}=-t \sum_{\langle i j\rangle \sigma} c_{i \sigma}^{\dagger} c_{j \sigma}
$$

where $c_{i \sigma}^{\dagger}$ creates an electron with spin $\sigma$ on the site $\mathbf{r}_{i}$ of the kagome lattice and $\langle i j\rangle$ denotes nearest neighbors. In momentum space Eq. (1) becomes $H_{0}=\sum_{\mathbf{k} \sigma} \Psi_{\mathbf{k} \sigma}^{\dagger} \mathcal{H}_{\mathbf{k}}^{0} \Psi_{\mathbf{k} \sigma}$ 
with $\Psi_{\mathbf{k} \sigma}=\left(c_{1 \mathbf{k} \sigma}, c_{2 \mathbf{k} \sigma}, c_{3 \mathbf{k} \sigma}\right)^{T}$ and

$$
\mathcal{H}_{\mathrm{k}}^{0}=-2 t\left(\begin{array}{ccc}
0 & \cos k_{1} & \cos k_{2} \\
\cos k_{1} & 0 & \cos k_{3} \\
\cos k_{2} & \cos k_{3} & 0
\end{array}\right) .
$$

The index $l=1,2,3$ in $c_{l \mathbf{k} \sigma}$ labels the three basis sites in the triangular unit cell; $\mathbf{a}_{1}=\hat{x}, \mathbf{a}_{2}=(\hat{x}+\sqrt{3} \hat{y}) / 2$ and $\mathbf{a}_{3}=\mathbf{a}_{2}-\mathbf{a}_{1}$ denote the three nearest neighbor vectors, and $k_{n}=\mathbf{k} \cdot \mathbf{a}_{n}$.

The spectrum of $\mathcal{H}_{\mathbf{k}}^{0}$, Fig. 1 1 , consists of one flat band $E_{\mathbf{k}}^{(3)}=2 t$ and two dispersive bands

$$
E_{\mathbf{k}}^{(1,2)}=t\left[-1 \pm \sqrt{4 A_{\mathbf{k}}-3}\right]
$$

with $A_{\mathbf{k}}=\cos ^{2} k_{1}+\cos ^{2} k_{2}+\cos ^{2} k_{3}$. Bands 1 and 2 touch at two inequivalent Dirac points $\mathbf{K}_{ \pm}=( \pm 2 \pi / 3,0)$ located at the corners of the hexagonal Brillouin zone (BZ), Fig. 1b. Bands 2 and 3 touch at the center of the BZ.

At $\frac{1}{3}$ filing the lowest band is filled and the low-energy electronic excitations of $H_{0}$ resemble those of graphene. To produce an insulator we now seek terms bi-linear in the electron operators that lead to the formation of a gap at the Dirac points. First, we focus on perturbations that do not further break the translational symmetry of $H_{0}$ and preserve $\mathcal{T}$. We have been able to identify two such terms: (i) a spin-independent lattice dimerization and (ii) a spin-orbit interaction induced hopping between the second neighbors [1, 15, 16. The former term breaks the inversion symmetry of the lattice while the latter breaks the $\mathrm{SU}(2)$ spin symmetry.

The dimerization is described by $H_{\mathrm{dim}}=$ $-\sum_{\langle i j\rangle \sigma} \delta t_{i j} c_{i \sigma}^{\dagger} c_{j \sigma}$, where $\delta t_{i j}= \pm \eta_{n}$ describes an alternating pattern of bond hopping integrals along the three principal spatial directions as illustrated in Fig. 1]d. In $k$-space this becomes

$$
\mathcal{H}_{\mathbf{k}}^{\operatorname{dim}}=2\left(\begin{array}{ccc}
0 & i \eta_{1} \sin k_{1} & i \eta_{2} \sin k_{2} \\
0 & i \eta_{3} \sin k_{3} \\
& 0
\end{array}\right),
$$

for both spin projections. (The lower triangle of the matrix is understood to be filled so that the matrix is hermitian.) The full expression for the spectrum of $\mathcal{H}_{\mathbf{k}}^{0}+\mathcal{H}_{\mathrm{k}}^{\text {dim }}$ is complicated but it is easy to see that a gap $\Delta_{\text {dim }} \simeq 2|\eta|$ with $\eta=\eta_{1}+\eta_{2}+\eta_{3}$ exists at the Dirac points.

The spin-orbit term takes form

$$
H_{\mathrm{SO}}=i \frac{2 \lambda}{\sqrt{3}} \sum_{\langle\langle i j\rangle\rangle \alpha \beta}\left(\mathbf{d}_{i j}^{1} \times \mathbf{d}_{i j}^{2}\right) \cdot \boldsymbol{\sigma}_{\alpha \beta} c_{i \alpha}^{\dagger} c_{j \beta},
$$

where $\lambda$ is the spin-orbit coupling strength, $\mathbf{d}_{i j}^{1,2}$ are nearest neighbor vectors traversed between second neighbors $i$ and $j$, and $\boldsymbol{\sigma}$ is the vector of Pauli spin matrices. Since $\mathbf{d}_{i j}^{1,2}$ all lie in the $x y$ plane in our $2 \mathrm{D}$ model, only $\sigma_{3}$ appears in Eq. (5) and the Hamiltonian decouples for the two spin projections along the $z$ axis. The pattern of spin-orbit induced second neighbor hoppings then resembles the Haldane model [15] and is illustrated in Fig. 11. In $k$-space one obtains

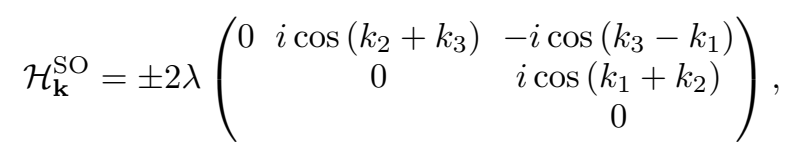

where the $+(-)$ sign refers to spin up (down) electrons. Once again, although the full spectrum is complicated it is easy to deduce that a gap $\Delta_{\mathrm{SO}}=4 \sqrt{3}|\lambda|$ opens up at the Dirac points. We remark that $H_{\mathrm{SO}}$ opens a gap also between bands 2 and 3 while $H_{\text {dim }}$ does not.

In order to develop some intuition for these insulating phases it is useful to examine the form of the low-energy Hamiltonians governing the excitations in the vicinity of the two Dirac points. This is obtained by linearizing $\mathcal{H}_{\mathbf{k}}=\mathcal{H}_{\mathbf{k}}^{0}+\mathcal{H}_{\mathbf{k}}^{\text {dim }}+\mathcal{H}_{\mathbf{k}}^{\text {SO }}$ near $\mathbf{K}_{ \pm}$and subsequently projecting onto the subspace associated with bands 1 and 2. Assuming that both $\eta$ and $\lambda$ are non-zero we obtain 4 independent Dirac Hamiltonians,

$$
h_{\mathbf{k} \ell \alpha}=v\left(\tau_{3} k_{x}+\tau_{1} k_{y}\right)+\tau_{2} m_{\ell \alpha},
$$

labeled by the spin index $\alpha= \pm$ and 'valley' index $\ell= \pm$. $\tau_{j}$ are Pauli matrices acting in the space spanned by the degenerate eigenstates of $\mathcal{H}_{\mathbf{K}_{+}}^{0}$ and $\mathcal{H}_{\mathbf{K}_{-}}^{0}, v=\sqrt{3} t$ is the Fermi velocity and

$$
m_{l \alpha}=2 \sqrt{3} \alpha \lambda+\ell \eta
$$

are Dirac masses whose relative signs define two distinct phases of the system. When $|\eta|>2 \sqrt{3}|\lambda|$ dimerization dominates and the Dirac masses at $\mathbf{K}_{+}$and $\mathbf{K}_{-}$exhibit opposite signs, independent of spin. When $|\eta|<2 \sqrt{3}|\lambda|$ the SO interaction dominates and the mass signs at the two Dirac points are the same for a given spin but differ for the opposite spin projections. The two phases meet at a pair of critical lines $|\eta|=2 \sqrt{3}|\lambda|$. When crossing these lines, two out of four gaps close and the associated Dirac masses change signs.

To understand the significance of the mass signs consider a boundary between the two phases, running along, say, the $x=0$ line in real space. For concreteness and simplicity we take $\lambda>0, \eta=0$ in the left half-plane and $\lambda=0, \eta>0$ in the right half-plane. Focusing first on the Dirac point $\mathbf{K}_{+}$, we note that the spin-up mass $m_{++}$ remains positive for all $x$, suggesting a fully gapped spectrum everywhere. The spin-down mass, however, necessarily undergoes a sign change across the $x=0$ boundary. Such a soliton mass profile is known to produce massless states [17] in the associated Dirac equation, localized near the boundary. Specifically, Dirac equation

$$
\left[v\left(-i \tau_{3} \partial_{x}-i \tau_{1} \partial_{y}\right)+\tau_{2} m(x)\right] \phi(x, y)=E \phi(x, y)
$$

with $m(x \rightarrow \pm \infty)= \pm m_{0}$ has a gapless solution

$$
\phi_{k}(x, y)=\left(\begin{array}{l}
1 \\
1
\end{array}\right) e^{i k y} e^{-\frac{1}{v} \int_{0}^{x} m\left(x^{\prime}\right) d x^{\prime}}
$$


extended along the boundary but localized in the transverse direction, with linearly dispersing energy $E_{k}=v k$. Similar analysis leads to a gapless state at node $\mathbf{K}_{-}$, but now for spin up and with $E_{k}=-v k$.

A pair of spin-filtered, oppositely dispersing gapless edge states is a hallmark of the topological spin-Hall insulator [1, 2, 3. The fact, apparent from the above construction, that these edge states depend only on the bulk band structure of the two insulators and not on the details of the edge indicates their topological origin. It follows that one of these phases must be a topological insulator. It is easy to see that the dimerized phase is smoothly connected to a trivial insulator. Consider increasing all $\eta$ 's continuously until $\eta_{1}=\eta_{2}=\eta_{3}=t$. At this point the kagome lattice breaks down into a collection of disconnected elementary triangles. This, clearly, is a trivial insulator. The spin-orbit phase, on the other hand, cannot be smoothly deformed into a trivial insulator and we show below by an explicit calculation that it indeed possesses a nontrivial $\mathrm{Z}_{2}$ invariant [1, 2, 3].

When a crystal possesses inversion symmetry the $Z_{2}$ topological invariant $\nu$ is easy to evaluate. According to Ref. [18] $\nu$ is related to the parity eigenvalues $\xi_{2 m}\left(\boldsymbol{\Gamma}_{i}\right)$ of the $2 m$-th occupied energy band at the four $\mathcal{T}$-invariant momenta $\boldsymbol{\Gamma}_{i}$. Our system is inversion symmetric when all $\eta$ 's vanish and so we can use this method to find $\nu$. If we select site 1 of the unit cell as the center of inversion then the parity operator acts as $\mathcal{P}\left[\psi_{1}(\mathbf{r}), \psi_{2}(\mathbf{r}), \psi_{3}(\mathbf{r})\right]=$ $\left[\psi_{1}(-\mathbf{r}), \psi_{2}\left(-\mathbf{r}-2 \mathbf{a}_{1}\right), \psi_{3}\left(-\mathbf{r}-2 \mathbf{a}_{2}\right)\right]$ on the triad of the electron wavefunctions in the unit cell labeled by vector r. In momentum space the parity operator becomes a diagonal $3 \times 3$ matrix $\mathcal{P}_{\mathbf{k}}=\operatorname{diag}\left(1, e^{-2 i \mathbf{a}_{1} \cdot \mathbf{k}}, e^{-2 i \mathbf{a}_{2} \cdot \mathbf{k}}\right)$. The four $\mathcal{T}$-invariant momenta in our system are marked in Fig. $1 \mathrm{~b}$ and can be expressed as $\boldsymbol{\Gamma}_{i}=\pi(\hat{x}+\hat{y} / \sqrt{3}) n_{i} / 2+$ $\pi(-\hat{x}+\hat{y} / \sqrt{3}) m_{i} / 2$ with $n_{i}, m_{i}=0,1$. It is straightforward to obtain the eigenstates of $\mathcal{H}_{\boldsymbol{\Gamma}_{i}}$ numerically and determine the parity eigenvalues of the occupied bands. We find that three $\xi$ 's are positive and one is negative. Which of the four $\xi$ 's is negative depends on the choice of the inversion center but the product $\Pi_{i} \xi\left(\boldsymbol{\Gamma}_{i}\right)=(-1)^{\nu}$ is independent of this choice and determines the non-trivial $\mathrm{Z}_{2}$ invariant $\nu=1$, confirming our hypothesis that the spin-orbit phase at $\frac{1}{3}$ filling is a topological insulator. Similar considerations for $\frac{2}{3}$ filling also yield $\nu=1$.

When the dimerization is present the inversion symmetry is broken and we must use the more general method of Ref. [1] to find $\nu$. This relies on counting the number of pairs of first order zeros of the quantity $P(\mathbf{k})=\operatorname{Pf}\left\langle u_{m \mathbf{k}}|\Theta| u_{n \mathbf{k}}\right\rangle$, where $u_{m \mathbf{k}}$ is the $m$-th eigenstate of $\mathcal{H}_{\mathbf{k}}, \Theta$ is the time-reversal operator and the Pfaffian is taken over occupied bands $m, n$. We obtain $u_{m \mathbf{k}}$ by the numerical diagonalization of $\mathcal{H}_{\mathbf{k}}$ and use it to straightforwardly evaluate $P(\mathbf{k})$. We find that the latter contains exactly one pair of first order zeros (located at $\mathbf{K}_{ \pm}$) in the SO phase when $|\eta|<2 \sqrt{3}|\lambda|$, indicating $\nu=1$. When $|\eta|>2 \sqrt{3}|\lambda|$ the zeros disappear and the system becomes a trivial insulator with $\nu=0$.

To further support our identification of the SO phase
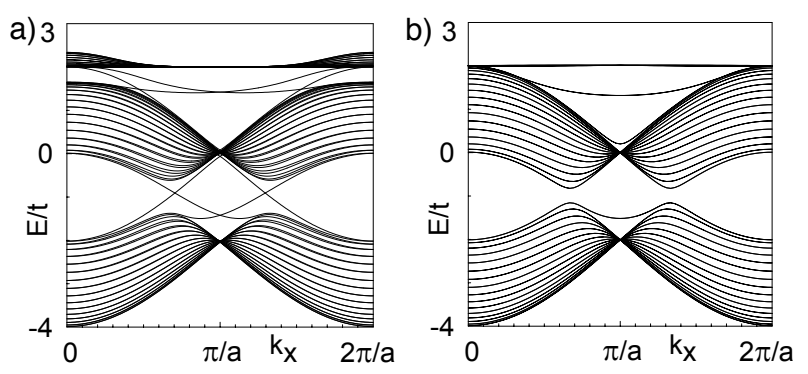

FIG. 2: Edge states in the lattice model for (a) spin-orbit and (b) dimerized insulator. A strip of width $N_{y}=16$ unit cells with open boundary conditions along $y$ and infinite along $x$ is used with $\lambda / t=0.1$ and $\eta_{1} / t=0.1$ for (a) and (b), respectively.

as a topological insulator we have performed numerical diagonalizations of the lattice Hamiltonians $H_{0}+H_{\text {dim }}$ and $H_{0}+H_{\text {SO }}$ using the strip geometry. In accord with the above arguments we find a pair of robust spin-filtered gapless states associated with each edge in the $\mathrm{SO}$ phase, Fig. 2 a, traversing the gap between bands 1 and 2 . A similar pair of states traverses the gap between bands 2 and 3 , confirming that the $\mathrm{SO}$ phase is a topological insulator at $\frac{1}{3}$ and $\frac{2}{3}$ filling. In the dimerized phase there are generically no such robust edge states, Fig. $2 \mathrm{~b}$, although for certain values of parameters and types of edges gapless edge states can occur. The latter mirror the edge states found along certain types of edges in graphene [19] but are not topological in character.

In graphene, a staggered on-site potential is known to open up a gap at the Dirac points [20]. We thus investigate the effect of the analogous CDW term,

$$
\mathcal{H}_{\mathbf{k}}^{\mathrm{CDW}}=\operatorname{diag}\left(\mu_{1}, \mu_{2}, \mu_{3}\right),
$$

on the kagome semi-metal, where $\mu_{l}$ represent the onsite potentials of the $l=1,2,3$ basis sites of the lattice, independent of spin. Repeating the procedure leading to Eq. (7) we find the low-energy Dirac Hamiltonians

$$
h_{\mathbf{k} \ell \alpha}=v\left[\tau_{3}\left(k_{x}-\mathcal{A}_{x}^{\ell}\right)+\tau_{1}\left(k_{y}-\mathcal{A}_{y}^{\ell}\right)\right]+\mathbb{1} \mu,
$$

for node $\ell$ and spin $\alpha$, with $\mu=\left(\mu_{1}+\mu_{2}+\mu_{3}\right) / 3$ and

$$
\begin{aligned}
& \mathcal{A}_{x}^{\ell}=\left(2 \mu_{3}-\mu_{1}-\mu_{2}\right) \ell / 6 \sqrt{3} t, \\
& \mathcal{A}_{y}^{\ell}=\left(\mu_{1}-\mu_{2}\right) \ell / 6 t .
\end{aligned}
$$

The CDW couples to the Dirac fermions as a gauge field. Owing to the factor $\ell$ in Eq. (13), the gauge potential has the opposite sign at the two Dirac points. Consequently, $\mathcal{A}$ must be thought of as an axial gauge field. This is consistent with the fact that the CDW does not break $\mathcal{T}$.

If we allow for additional fermion bi-linears that break the translational symmetry of $H_{0}$ then many other insulating phases become possible. Of these, we briefly mention but one that realizes the analog of the Kekulé 
phase in graphene [14. This occurs for a perturbation with a wavevector spanning the two nodal points $\mathbf{K}_{+}$and $\mathbf{K}_{-}$in Fig. 1p. In the linearized theory such a perturbation leads to a Dirac mass that is off-diagonal in the space of nodal Hamiltonians and is in general complexvalued. In graphene, vortices in such a complex mass are known to carry a fractional charge $\pm e / 2$ (assuming spinless electrons) 14 and obey fractional exchange statistics [21]. On the kagome lattice one possible realization of such a perturbation is the 'trimerization' depicted in Fig. 11. The unit cell contains 9 atoms and there are 3 distinct degenerate ground states related to the one depicted by translations through $2 \mathbf{a}_{1}$ and $2 \mathbf{a}_{2}$. We have verified that this perturbation indeed opens up a gap at the Dirac points and the three patterns produce offdiagonal Dirac masses with different complex phases. In analogy with the domain wall in the trimerized 1D chain 22 we expect the vortex in the complex mass to bind fractional charge $\pm e / 3, \pm 2 e / 3$. This is also suggested by the Goldstone-Wilczek type counting argument 23 . generalized to 2 spatial dimensions 24 that can be constructed for the kagome lattice 25]. We leave the details of this and other interesting related problems, such as the exchange statistics of these objects, to future study.

A question naturally arises as to the experimental realization of a system of itinerant electrons on the kagome lattice that would support the exotic phenomena predicted above. Copper atoms in $\mathrm{ZnCu}_{3}(\mathrm{OH})_{6} \mathrm{Cl}_{2}$, known as Herbertsmithite [26, and iron atoms in Jarosites [27, provide good model Heisenberg kagome antiferromag- nets. The kagome lattice has also been argued to play a role in transport and magnetic properties of layered cobalt oxides, such as $\mathrm{Na}_{x} \mathrm{CoO}_{2}$ [28]. We hope that our theoretical findings will provide motivation for future experimental searches into possible realizations of the kagome lattice with itinerant electrons close to $\frac{1}{3}$ or $\frac{2}{3}$ filling. We note that it might be possible to artificially engineer Hamiltonian (1) by modulating the twodimensional electron gas with a periodic potential with kagome symmetry, as recently demonstrated for 'artificial graphene' 29].

When the basic tight-binding Hamiltonian is at hand the additional terms required to form an insulator can come about in various ways. In a crystal the SO coupling arises naturally. In graphene the relevant coupling strength is too small to open up a significant gap but in a lattice made of heavier ions $\lambda$ will be larger. The perturbations considered above can also arise as interactiondriven instabilities of itinerant electrons on the lattice. For the Dirac semi-metal at $\frac{1}{3}$ filling a finite interaction strength $U$ (of order $t$ ) is needed to open up a gap 30 . At $\frac{2}{3}$ filling, however, the band crossing is quadratic and the instability towards the insulating phase occurs at infinitesimal (repulsive) interaction [31, making such system a promising candidate for a $2 \mathrm{D}$ topological insulator.

Acknowledgment.- Authors are indebted to T. Aitken, G. Rosenberg, B. Seradjeh and C. Weeks for stimulating discussions. Support for this work came from NSERC, CIfAR and The China Scholarship Council.
[1] C. L. Kane, and E. J. Mele, Phys. Rev. Lett. 95, 146802 (2005).

[2] L. Fu, C. L. Kane, and E. J. Mele, Phys. Rev. Lett. 98 106803 (2007).

[3] J. E. Moore and L. Balents, Phys. Rev. B 75 121306(R) (2007).

[4] R. Roy, Phys. Rev. B 79, 195322 (2009).

[5] X.-L. Qi, T.L. Hughes, and S.-C. Zhang, Phys. Rev. B 78, 195424 (2008).

[6] A.M. Essin, J.E. Moore, D. Vanderbilt, Phys. Rev. Lett. 102, 146805 (2008).

[7] X.-L. Qi, R. Li, J. Zang, S.-C. Zhang, Science 323, 1184 (2009).

[8] L. Fu and C. L. Kane, Phys. Rev. Lett. 100, 096407 (2008).

[9] M. König et al., Science 318, 766 (2007).

[10] D. Hsieh et al., Nature 452, 970 (2008).

[11] Y. Xia et al., arXiv:0812.2078

[12] J. C. Y. Teo, L. Fu, and C. L. Kane, Phys. Rev. B 78 045426 (2008).

[13] H.-J. Zhang et al., Phys. Rev. B 80085307 (2009).

[14] C.-Y. Hou, C. Chamon, and C. Mudry, Phys. Rev. Lett. 98, 186809 (2007).

[15] F.D.M. Haldane, Phys. Rev. Lett. 61, 2015 (1988).

[16] C. L. Kane, and E. J. Mele, Phys. Rev. Lett. 95, 226801 (2005).

[17] R. Jackiw and C. Rebbi, Phys. Rev. D 13, 3398 (1976).
[18] L. Fu and C. L. Kane, Phys. Rev. B 76, 045302 (2007).

[19] K. Nakada, M. Fujita, G. Dresselhaus and M.S. Dresselhaus, Phys. Rev. B 54, 17954 (1996).

[20] G.W. Semenoff, Phys. Rev. Lett. 53, 2449 (1984).

[21] B. Seradjeh and M. Franz, Phys. Rev. Lett. 101, 146401 (2008).

[22] W.P. Su and J.R. Schrieffer, Phys. Rev. Lett. 46, 738 (1981).

[23] J. Goldstone and F. Wilczek, Phys. Rev. Lett. 47, 986 (1981).

[24] B. Seradjeh, C. Weeks and M. Franz, Phys. Rev. B 77, 033104 (2008).

[25] M. Franz, (unpublished).

[26] J.S. Helton, et al. Phys. Rev. Lett. 98, 107204 (2007).

[27] A.S. Wills, A. Harrison, C. Ritter and R.I. Smith, Phys. Rev. B 61, 6156 (2000).

[28] W. Koshibae and S. Maekawa, Phys. Rev. Lett. 91, 257003 (2003).

[29] M. Gibertini, et al., Phys. Rev. B 79, 241406(R) (2009).

[30] S. Raghu, X.-L. Qi, C. Honerkamp and S.-C. Zhang, Phys. Rev. Lett. 100, 156401 (2008).

[31] K. Sun, H. Yao, E. Fradkin and S.A. Kivelson, Phys. Rev. Lett. 103, 046811 (2009). 